\title{
Anisotropic impurities in anisotropic superconductors
}

\author{
Miodrag L. Kulić ${ }^{1}$ and Oleg V. Dolgov ${ }^{2}$ \\ ${ }^{1}$ Physikalisches Institut, Universität Bayreuth, 95440 Bayreuth, \\ Germany \\ ${ }^{2}$ Institut für Theoretische Physik, Universität Tübingen, \\ Germany \\ (today)
}

\begin{abstract}
Physical properties of anisotropic superconductors like the critical temperature and others depend sensitively on the electron mean free path. The sensitivity to impurity scattering and the resulting anomalies are considered a characteristic feature of strongly anisotropic pairing. These anomalies are usually analyzed in terms of s-wave impurity scattering which leads to universal pair breaking effects depending on only two scattering parameters, the mean free path and the impurity cross section. We investigate here corrections coming from anisotropies in the scattering cross section, and find not only quantitative but also qualitative deviations from universal s-wave isotropic pairbreaking. The properties we study are the transition temperature, the density of states, quasiparticle bound states at impurities, and pinning of flux lines by impurities.
\end{abstract}

PACS. 74.20.-z, 74.20.Fg 


\section{INTRODUCTION}

Unconventional anisotropic pairing is evidently realized in high temperature superconductors $(H T S)$ - see review1, and probably in some heavy Fermion superconductors $(H F S)$ - see review日. There are good evidences for d-wave pairing in optimally doped HTS oxides 1 but the type of pairing in HFS is still uncleart. The effect of impurities on unconventional pairing is an important tool in analyzing the symmetry of the pairing amplitude, and is the subject of a number of experimental and theoretical works, 1 . Most of calculations were done assuming an s-wave impurity scattering potential $u_{i m p}\left(\mathbf{p}, \mathbf{p}^{\prime}\right)=$ const, and taking either the Born limit $\left(N(0) u_{i m p} \ll 1\right)$ or unitarity limit $\left(N(0) u_{i m p} \gg 1\right)$.

Surprisingly, a number of experiments on the optimally HTS oxides have shown that $d-$ wave pairing is quite robust, i.e. not very sensitive to various kinds of impurities and defects. For instance, the decrease of the critical temperature $T_{c}\left(\rho_{i m p}\right)$, with increasing residual resistivity, $\rho_{i m p}$, is much smaller than the theory with the $s$ - wave impurity scattering predicts, 0 , 3. A way out of this experimental and theoretical discrepancy of pair-breaking effects by impurities in HTS oxides was proposed by the authors of Refs.0 0 , who invoked a momentum dependent impurity scattering potential with an appreciable contribution in the d-channel. The microscopic theory in Ref. potential by strong correlations, which gives rise to a pronounced forward scattering peak, while backward scattering is suppressed, as first proposed in Ref. of this theory to impurity scattering 8 shows that in addition to the contribution in the s-channel there is a significant contribution to the Born amplitude from the d-channel of the same magnitude, in particular for low (hole) doping concentration, $\delta<0.2$. As a consequence, the decrease of $T_{c}\left(\rho_{i m p}\right)$ with increasing $\rho_{i m p}$ is much slower than the theory with exclusively s-wave impurity scattering predicts]. This renormalization effect explains the robustness of d-wave pairing in HTS oxides.

One may rise the question whether this robustness also holds far away from $T_{c}$ and for very strong scattering potential, for instance in the unitarity limit. To answer this question 
we shall analyze a class of models by calculating the scattering $T$-matrix with an impurity potential that depends on the scattering angles.

A related class of problems, which we study in section III, is related to the impurity scattering in the two-band model. Recently, several models for the pairing mechanism in HTS oxides based on two-band and multi-band models $1,10,11,12$ were suggested, and impurity effects studied in Born approximation. Magnetic and non-magnetic interband scattering can lead in this model to a lowering of the critical temperature and also to a relative sign change of the order parameters in different band\$11. In section III we analyze the changes in the two-band model when going beyond the Born limit. It was shown that in the unitarity limit the Anderson theorem holds.

In previous sections we studied a homogeneous superconductor with homogeneously distributed impurities. Selected inhomogeneous problems are studied in section IV, such as the bound states at an impurity, and the pinning energy at an impurity (defect) of singly- and double-quantized vortices.

\section{ANISOTROPIC SCATTERING IN ANISOTROPIC AND HOMOGENEOUS SUPERCONDUCTORS}

In the following we analyze superconducting properties of anisotropic superconductors in the presence of momentum-dependent nonmagnetic impurity scattering by the quasiclassical equations of Eilenberger, Larkin-Ovchinnikov13,14 (ELO equations ). For a homogeneous distribution the quasiclassical Green's function matrix, $\hat{g}\left(\mathbf{p}_{F}, \mathbf{R}, \omega_{n}\right)$, is independent of $\mathbf{R}$, and the quasiclassical equations read

$$
\begin{gathered}
{\left[i \omega_{n} \hat{\tau}_{3}-\hat{\Delta}\left(\mathbf{p}_{F}, \omega_{n}\right)-\hat{\sigma}_{i m p}\left(\mathbf{p}_{F}, \omega_{n}\right), \hat{g}\left(\mathbf{p}_{F}, \omega_{n}\right)\right]=0} \\
\hat{g}^{2}\left(\mathbf{p}_{F}, \omega_{n}\right)=-\hat{1}
\end{gathered}
$$

We assume weak-coupling superconductivity with $\hat{\Delta}\left(\mathbf{p}_{F}\right)\left(=i \Delta\left(\mathbf{p}_{F}\right) \hat{\tau}_{2}\right)$, where $\Delta\left(\mathbf{p}_{F}\right)$ is real. The $2 \times 2$ matrices $\hat{\tau}_{0} \equiv \hat{1}$ and $\hat{\tau}_{1,2,3}$ are Nambu-Gor'kov matrices. The effect of 
nonmagnetic impurities is described by the self-energy, $\hat{\sigma}_{i m p}$, given in terms of the forward scattering part, $\hat{t}\left(\mathbf{p}_{F}, \mathbf{p}_{F}^{\prime}, \omega_{n}\right)$, of the T-matrix 13 , 4 , 15

$$
\hat{\sigma}_{i m p}\left(\mathbf{p}_{F}, \omega_{n}\right)=c \hat{t}\left(\mathbf{p}_{F}, \mathbf{p}_{F}, \omega_{n}\right)
$$

where $c(\ll 1)$ is the impurity concentration.

For simplicity we assume an isotropic Fermi surface but pairing and impurity scattering are angle-dependent, i.e. $\Delta\left(\mathbf{p}_{F}\right) \equiv \Delta(\mathbf{s}), \hat{g}\left(\mathbf{p}_{F}, \omega_{n}\right) \equiv \hat{g}(\mathbf{s}, n)$ and $\hat{t}\left(\mathbf{p}_{F}, \mathbf{p}_{F}^{\prime}, \omega_{n}\right) \equiv \hat{t}\left(\mathbf{s}, \mathbf{s}^{\prime}, n\right)$ where $\mathbf{s}=\mathbf{p}_{F} / p_{F}$. The T-matrix is the solution of the equation

$$
\hat{t}\left(\mathbf{s}, \mathbf{s}^{\prime}, n\right)=u\left(\mathbf{s}, \mathbf{s}^{\prime}\right) \hat{1}+N(0) \int d \mathbf{s}^{\prime \prime} u\left(\mathbf{s}, \mathbf{s}^{\prime \prime}\right) \hat{g}\left(\mathbf{s}^{\prime \prime}, n\right) \hat{t}\left(\mathbf{s}^{\prime \prime}, \mathbf{s}^{\prime}, n\right),
$$

where for the $2 D$ systems, which we consider here, one has $\int d \mathbf{s}\{..\} \equiv \int\{.\}. d \theta / 2 \pi$. Since $\Delta(\mathbf{s})$ is real one has $\hat{g}=g_{2} \hat{\tau}_{2}+g_{3} \hat{\tau}_{3}$ and $\hat{t}$ is given by $\hat{t}=t_{0} \hat{\tau}_{0}+t_{1} \hat{\tau}_{1}+t_{2} \hat{\tau}_{2}+t_{3} \hat{\tau}_{3}$.

Because the unperturbed solution has the form $\left(\omega_{n}=\pi T(2 n+1)\right)$

$$
\hat{g}^{(0)}(\mathbf{s}, n)=-\frac{i \omega_{n} \hat{\tau}_{3}-i \Delta_{0}(\mathbf{s}) \hat{\tau}_{2}}{\sqrt{\omega_{n}^{2}+\Delta_{0}^{2}(\mathbf{s})}}
$$

then $\hat{g}(\mathbf{s}, n)$ is searched for in the form

$$
\hat{g}(\mathbf{s}, n)=-\frac{i \tilde{\omega}_{n}(\mathbf{s}) \hat{\tau}_{3}-i \tilde{\Delta}\left(\mathbf{s}, \omega_{n}\right) \hat{\tau}_{2}}{\sqrt{\tilde{\omega}_{n}^{2}(\mathbf{s})+\tilde{\Delta}^{2}\left(\mathbf{s}, \omega_{n}\right)}},
$$

where

$$
\begin{gathered}
\tilde{\omega}_{n}(\mathbf{s})=\omega_{n}(\mathbf{s})+i c_{i} t_{3}(\mathbf{s}, \mathbf{s}, n) \\
\tilde{\Delta}\left(\mathbf{s}, \omega_{n}\right)=\Delta(\mathbf{s})-i c_{i} t_{2}(\mathbf{s}, \mathbf{s}, n) .
\end{gathered}
$$

The self-consistency equation for $\Delta(\mathbf{s})$ is given by

$$
\Delta(\mathbf{s})=N(0) T \sum_{n} \int d \mathbf{s}^{\prime} V\left(\mathbf{s}, \mathbf{s}^{\prime}\right) g_{2}\left(\mathbf{s}^{\prime}, n\right)
$$

where the pairing potential $V_{p}\left(\mathbf{s}, \mathbf{s}^{\prime}\right)=V_{p} Y(\mathbf{s}) Y\left(\mathbf{s}^{\prime}\right)$ is assumed in the factorized form with $<Y^{2}(\mathbf{s})>_{\mathbf{s}}=1$. The latter implies that the order parameter has the form $\Delta(\mathbf{s})=\Delta \cdot Y(\mathbf{s})$. 
For convenience we define $\Gamma_{u}\left(\equiv c \gamma_{u}\right)=c(\pi N(0))^{-1}$ and $v\left(\mathbf{s}, \mathbf{s}^{\prime}\right) \equiv \pi N(0) u\left(\mathbf{s}, \mathbf{s}^{\prime}\right)$. In what follows we consider the effects of anisotropic impurity scattering on the anisotropic pairing where $<Y(\mathbf{s})>_{\mathbf{s}}=0$.

\section{Anisotropic impurity scattering and nodeless anisotropic pairing}

First, we consider the nodeless d-wave like pairing $\Delta(\mathbf{s})=\Delta \cdot Y(\mathbf{s})$ which is characterized by $\langle Y(\mathbf{s})\rangle_{\mathbf{s}}=0$ and $Y^{2}(\mathbf{s})=1$. This means that there is a finite gap everywhere on the Fermi surface, i.e. $\Delta(\mathbf{s}) \neq 0$. It is interesting to mention, that besides the simplicity of this kind of pairing and its adequacy in some qualitative understanding of d-wave pairing it also appears to be a solution of the spin-bag mode 16 for HTS oxides. In this model the nodeless $d$-wave like pairing is due to residual (longitudinal and transverse) spin fluctuations on the antiferromagnetic background, where the $A F$ order is distorted locally by hole doping and the spin-bag is formed around doped holes. The impurity scattering potential is assumed to have the form

$$
v\left(\mathbf{s}, \mathbf{s}^{\prime}\right)=v_{0}+v_{2} Y(\mathbf{s}) Y\left(\mathbf{s}^{\prime}\right),
$$

i.e. it contains an anisotropic contribution in the same channel as the unconventional pairing. The solution of Eq.(四) for $t_{3}$ and $t_{2}$ is searched in the form

$$
\begin{gathered}
t_{3}\left(\mathbf{s}, \mathbf{s}^{\prime}\right)=\left[\tilde{t}_{30}(n)+\tilde{t}_{32}(n) Y(\mathbf{s}) Y\left(\mathbf{s}^{\prime}\right)\right] g_{3}, \\
t_{2}\left(\mathbf{s}, \mathbf{s}^{\prime}\right)=\tilde{t}_{2}(n)\left[g_{2}(\mathbf{s}, n)+g_{2}\left(\mathbf{s}^{\prime}, n\right)\right] .
\end{gathered}
$$

(Note, in this model $g_{2}(\mathbf{s}, n)=\tilde{g}_{2}(n) Y(\mathbf{s}), g_{2}^{2}(\mathbf{s}, n)=\tilde{g}_{2}^{2}(n), g_{3}(\mathbf{s}, n)=g_{3}(n)$ and due to $E q$ (2) one has $g_{3}^{2}(\mathbf{s}, n)+\tilde{g}_{2}^{2}(\mathbf{s}, n)=-1$.) The solution is given by

$$
\begin{aligned}
& \tilde{t}_{30}(n)=\gamma_{u} v_{0}^{2} \frac{1+v_{2}^{2}}{\left(1+v_{0}^{2}\right)\left(1+v_{2}^{2}\right)+\left(v_{0}-v_{2}\right)^{2} \tilde{g}_{2}^{2}(n)}, \\
& \tilde{t}_{2}(n)=\gamma_{u} v_{0} v_{2} \frac{1+v_{0} v_{2}}{\left(1+v_{0}^{2}\right)\left(1+v_{2}^{2}\right)+\left(v_{0}-v_{2}\right)^{2} \tilde{g}_{2}^{2}(n)},
\end{aligned}
$$

while $\tilde{t}_{32}(n)=\tilde{t}_{30}\left(n, v_{0} \leftrightarrow v_{2}\right)$. Several interesting results comes out in this case. 
(a) The critical temperature $T_{c}$

In the limit $T \rightarrow T_{c}$ Eqs.(19, 13- 14) become

$$
\ln \frac{T_{c}}{T_{c 0}}=\Psi\left(\frac{1}{2}\right)-\Psi\left(\frac{1}{2}+\frac{\Gamma_{p b}}{2 \pi T_{c}}\right),
$$

where the pair-breaking parameter $\Gamma_{p b}$ is given by $\left(\Gamma_{u}=c \pi / N(0)\right)$

$$
\Gamma_{p b}=\Gamma_{u} \frac{\left(v_{0}-v_{2}\right)^{2}}{\left(1+v_{0}^{2}\right)\left(1+v_{2}^{2}\right)} .
$$

Note that $T_{c}$ vanishes for $\Gamma_{p b}^{c} \approx 0.88 T_{c 0}$ and this pairing is in some respects similar to dwave pairing. It is apparent from Eqs.(15-16) that the pair-breaking effect of impurities is weakened in the presence of momentum-dependent scattering and it is even zero for $v_{0}=v_{2}$. The latter result has been previously derived in the Born approximation 5 . For $v_{2} \approx v_{0}$ the slope $d T_{c} / d \rho_{i m p}$ can be very small even for appreciable values of $\rho_{i m p} \sim \Gamma_{t r}=\Gamma_{u}\left(\bar{\sigma}_{0}+\bar{\sigma}_{2}\right)$, because in that case $\Gamma_{p b} \ll \Gamma_{t r}$ as indicate the experimental results ofl. The parameters $\bar{\sigma}_{i}$ are given by

$$
\bar{\sigma}_{i}=\frac{v_{i}^{2}}{1+v_{i}^{2}}, \quad i=0,1,2 \ldots
$$

The resistivity, $\rho_{i m p}$, and the reduction of $T_{c}$ due to impurity scattering, $T_{c}\left(\rho_{i m p}\right)$, depend on the classical transition rate, $W\left(\mathbf{s}, \mathbf{s}^{\prime}\right)=\Gamma_{u}\left|t_{N}\left(\mathbf{s}, \mathbf{s}^{\prime}, n\right)\right|^{2}$ in the normal state 17 . This transition rate comprises all the needed information on impurity scattering for either solving the normal state Boltzmann equation to determine $\rho_{i m p}$ or the linearized gap equation Eq.(9) to determine $T_{c}$. For the latter purpose one needs linear (integral) equation for $g_{2}(\mathbf{s}, n)$ which reads

$$
\left|\omega_{n}\right| g_{2}(\mathbf{s}, n)-\Delta(\mathbf{s})+\int d \mathbf{s}^{\prime} W\left(\mathbf{s}, \mathbf{s}^{\prime}\right)\left[g_{2}(\mathbf{s}, n)-g_{2}\left(\mathbf{s}^{\prime}, n\right)\right]=0,
$$

where the normal state t-matrix $t_{N}\left(\mathbf{s}, \mathbf{s}^{\prime}, n\right)$ is the solution of the equation

$$
t_{N}\left(\mathbf{s}, \mathbf{s}^{\prime}, n\right)=v\left(\mathbf{s}, \mathbf{s}^{\prime}\right)-i \pi \operatorname{sign}\left(\omega_{n}\right) \int d \mathbf{s}^{\prime \prime} v\left(\mathbf{s}, \mathbf{s}^{\prime \prime}\right) t_{N}\left(\mathbf{s}^{\prime \prime}, \mathbf{s}^{\prime}, n\right) .
$$


Hence, measurements of $T_{c}\left(\rho_{i m p}\right)$ curve carry not enough informations on the microscopic scattering data, i.e. the scattering T-matrix. More such informations are contained in spectroscopic data on anisotropic superconductors at temperatures $T \ll T_{c}$, such as tunneling data or optical data at about gap frequency.

(b) The density of states, $N(\omega)=N(0) \operatorname{Im} \int d \mathbf{s} g_{3}\left(\mathbf{s}, i \omega_{n} \rightarrow \omega-i \eta\right)$, depends in the presence of pair-breaking impurities significantly on the values $v_{0}$ and $v_{1}$. It is known 18 that in the case of s-wave scattering only $\left(v_{2}=0\right)$ one has $N(\omega=0) \neq 0$ for $\Gamma_{u} v_{0}^{2}>\Delta$, and the highest value, $N(\omega=0)=N(0) /\left[0.5+0.5\left(1+(2 \Delta / \Gamma)^{2}\right)^{1 / 2}\right]^{1 / 2}$, where $\Gamma \equiv \Gamma_{u} \bar{\sigma}_{0}$, is reached in the unitarity limit. On the other hand, one obtains in the limiting case, $v_{0}=v_{2}$, a restoration of the gap, $N(\omega=0)=0$. Despite of the strong scattering limit $N(\omega)$ is BCS-like.

\section{Isotropic impurity scattering and $d$ - wave pairing}

Let us study a two-dimensional superconductor with the pairing function $\Delta(\mathbf{s}) \equiv \Delta(\varphi)(=$ $\left.\Delta \cdot Y_{2}(\varphi)\right) \sim \cos 2 \varphi-d-$ wave pairing. Note this case seems to be more realistic for HTS oxides than the previous one, because the " $\cos 2 \varphi$ " pairing has nodes at the simply connected Fermi surface. We assume that the isotropic impurity potential depends on the transferred scattering angle

$$
v\left(\varphi, \varphi^{\prime}\right)=v_{0}+2 v_{1} \cos \left(\varphi-\varphi^{\prime}\right)+2 v_{2} \cos 2\left(\varphi-\varphi^{\prime}\right)
$$

where $v\left(\varphi, \varphi^{\prime}\right)$ contains the pairing channel $\left(\sim Y_{2}(\varphi) Y_{2}\left(\varphi^{\prime}\right)\right)$ too. (This problem but with $v_{2}=0$ is studied in 19 but there is an inappropriate sign in the $t_{2}$-matrix, which in fact corresponds to a magnetic impurity scattering). From Eqs.(13 - 14) one obtains the pairbreaking parameter $\Gamma_{p b}$

$$
\Gamma_{p b}=\Gamma_{u}\left[\bar{\sigma}_{0} \frac{(1-\alpha)^{2}+\alpha^{2}\left(1+v_{0}^{2}\right)}{1+\alpha^{2} v_{0}^{2}}+\bar{\sigma}_{1}\right],
$$

where $\alpha=v_{2} / v_{0}$ and $\bar{\sigma}_{i}$ are given by Eq.(17).

In order to analyze $T_{c}\left(\rho_{i m p}\right)$ dependence, where the residual impurity resistivity $\rho_{i m p} \sim$ $\Gamma_{t r}$, we need the transport scattering $\Gamma_{t r}$ which is in this case given by 


$$
\Gamma_{t r}=\Gamma_{u}\left\{\bar{\sigma}_{0}^{2}+2 \bar{\sigma}_{1}^{2}+2 \bar{\sigma}_{2}^{2}-2 \bar{\sigma}_{0}\left[\bar{\sigma}_{1}\left(1+\frac{1}{v_{0} v_{1}}\right)+\bar{\sigma}_{2}\left(1+\frac{1}{v_{0} v_{2}}\right)\right]\right\}
$$

If one wants to interpret depairing effects of impurities and robustness of pairing in HTS oxides in terms of the above results then the experiments $\mathrm{B}$ imply that the ratio, $\Gamma_{p b} / \Gamma_{t r}$, should be minimum. In the case when $v_{2} \ll v_{1}$ one obtains, $\Gamma_{p b} / \Gamma_{t r}=2$, in both, the Born and unitarity, $v_{0}, v_{1} \rightarrow \infty$, limits. For, $v_{1} \ll v_{2}$, the pair-breaking parameter, $\Gamma_{p b}$, is minimized for $\alpha=1 / 2$ which gives, $\Gamma_{p b} / \Gamma_{t r} \approx 1 / 3$, in both limits. This means that the latter case is more appropriate candidate, than the case, $v_{2} \ll v_{1}$, for the qualitative explanation of robustness of d-wave pairing in $H T S$ oxides.

\section{TWO-BAND MODEL WITH NONMAGNETIC IMPURITIES}

The interest in two(multi)-band models and in the impurity effects is renewed after the

discovery of $H T S$ oxides, $10,12,11$, where various kinds of the intra- and inter-band pairing and impurity scattering are considered. By assuming only intra-band pairing $\Delta_{\alpha}(\alpha=1,2)$ the effect of the nonmagnetic impurities in the Born approximation is described by the equations ( $n$ enumerates Matsubara frequencies)

$$
\begin{gathered}
\tilde{\omega}_{\alpha n}=\omega_{n}+\sum_{\beta} \frac{\tilde{\omega}_{\beta n}}{2 Q_{\beta n}} \gamma_{\alpha \beta} \\
\tilde{\Delta}_{\alpha n}=\Delta_{\alpha}+\sum_{\beta} \frac{\tilde{\Delta}_{\beta n}}{2 Q_{\beta n}} \gamma_{\alpha \beta} \\
\Delta_{\alpha}=\pi T \sum_{\beta, n}^{-\omega_{D}<\omega_{n}<\omega_{D}} \lambda_{\alpha \beta} \frac{\tilde{\Delta}_{\beta n}}{Q_{\beta n}},
\end{gathered}
$$

where $Q_{\alpha n}=\sqrt{\tilde{\omega}_{\alpha n}^{2}+\tilde{\Delta}_{\alpha n}^{2}}, \gamma_{\alpha \beta}=u_{\alpha \beta}^{2} N_{\beta}(0)$ for the nonmagnetic impurity scattering and $\lambda_{\alpha \beta}=V_{\alpha \beta}^{p} N_{\beta}(0)$ are corresponding coupling constants. In Ref.11 are considered various possibilities for the suppression of the critical temperature, as well as the relative sign of $\Delta_{1}$ and $\Delta_{2}$, in the Born limit for nonzero values of $\lambda_{\alpha \beta}$ and $\gamma_{\alpha \beta}$. We note some interesting conclusions obtained in Born approximation which shall be compared with the results 
obtained in the unitarity limit: $(i)$ the diagonal scattering rate $\gamma_{11}$ and $\gamma_{22}$ disappear from the linearized Eq.(25) for $T_{c} ;(i i)$ in the case $\lambda_{11} \neq 0, \lambda_{22}=\lambda_{12}=\lambda_{21}=0$ the depression of $T_{c}$ is given by $\delta T_{c} / T_{c}=-\pi \gamma_{12} / 8 T_{c}$; $(i i)$ for $\lambda_{11}=\lambda_{22} \neq 0$ and $\lambda_{12}=\lambda_{21}=\lambda_{\perp}<0$ one has $\operatorname{sign}\left(\Delta_{1} / \Delta_{2}\right)=-1$ and $\delta T_{c} / T_{c}=-\pi\left(\gamma_{12}+\gamma_{21}\right) / 8 T_{c}$, while the sign of $\Delta_{1}$ and $\Delta_{2}$ is unchanged by the impurities.

The $t$-matrix equation in the two-band model has the form $(\alpha, \beta, \gamma=1,2)$ ( we consider a rather small impurity concentration and neglect an interband hybridization)

$$
\hat{\mathbf{t}}(n)=\hat{\mathbf{u}}+\sum_{\gamma} \hat{\mathbf{u}} \mathbf{N}(0) \hat{\mathbf{g}}(n) \hat{\mathbf{t}}(n)
$$

where $\hat{\mathbf{t}}(n)=\sum_{i=0}^{3} \mathbf{t}_{i} \otimes \hat{\tau}_{i}, \hat{\mathbf{g}}(n)=\mathbf{g}_{3} \otimes \hat{\tau}_{3}+\mathbf{g}_{2} \otimes \hat{\tau}_{2}$ and $\otimes$ is the direct product of matrices in the band space (bold) and in the Nambu space (hat). $\mathbf{g}_{2}, \mathbf{g}_{3}$ and $\mathbf{N}(0)$ are diagonal matrices in the band space.

In the case of nonmagnetic impurities one has $\hat{\mathbf{u}}^{N}=\mathbf{u}^{N} \otimes \hat{\tau}_{0}$ and since $\mathbf{g}_{1}=0$ one has

$$
\begin{gathered}
\mathbf{t}_{0}^{N}(n)=\mathbf{u}^{N}+\mathbf{u}^{N} \mathbf{N}(0)\left[\mathbf{g}_{3}(n) \mathbf{t}_{3}^{N}(n)+\mathbf{g}_{3}(n) \mathbf{t}_{3}^{N}(n)\right] \\
\mathbf{t}_{1}^{N}(n)=\mathbf{u}^{N} \mathbf{N}(0)\left[-i \mathbf{g}_{3}(n) \mathbf{t}_{2}^{N}(n)+i \mathbf{g}_{2}(n) \mathbf{t}_{3}^{N}(n)\right] \\
\mathbf{t}_{2}^{N}(n)=\mathbf{u}^{N} \mathbf{N}(0)\left[i \mathbf{g}_{3}(n) \mathbf{t}_{1}^{N}(n)+\mathbf{g}_{2}(n) \mathbf{t}_{0}^{N}(n)\right] \\
\mathbf{t}_{3}^{N}(n)=\mathbf{u}^{N} \mathbf{N}(0)\left[\mathbf{g}_{3}(n) \mathbf{t}_{0}^{N}(n)-i \mathbf{g}_{2}(n) \mathbf{t}_{1}^{N}(n)\right]
\end{gathered}
$$

Let us consider for simplicity the case when $u_{11}^{N}, u_{22}^{N}=0$ but interband scattering, $u_{12}^{N}=$ $u_{21}^{N}=u \neq 0$, and introduce three parameters

$$
\sigma=\frac{\pi^{2} N_{1}(0) N_{2}(0) u^{2}}{1+\pi^{2} N_{1}(0) N_{2}(0) u^{2}}
$$

and

$$
\Gamma_{i}=\frac{c \cdot \sigma}{\pi N_{i}(0)}, \quad i=1,2
$$


After some straightforward calculations one obtains the renormalized frequencies, $\tilde{\omega}_{i n}$, and order parameters $\tilde{\Delta}_{i n}$

$$
\begin{gathered}
\tilde{\omega}_{1 n}=\omega_{n}+\Gamma_{1} \frac{(\sigma-1)\left(\tilde{\omega}_{1 n}^{2}+\tilde{\Delta}_{1 n}^{2}\right) \tilde{\omega}_{2 n}-\sigma \tilde{\omega}_{1 n} \sqrt{\tilde{\omega}_{1 n}^{2}+\tilde{\Delta}_{1 n}^{2}} \sqrt{\tilde{\omega}_{2 n}^{2}+\tilde{\Delta}_{2 n}^{2}}}{\operatorname{det} 1} \\
\tilde{\Delta}_{1 n}=\Delta_{1}+\Gamma_{1} \frac{(\sigma-1)\left(\tilde{\omega}_{1 n}^{2}+\tilde{\Delta}_{1 n}^{2}\right) \tilde{\Delta}_{2 n}-\sigma \tilde{\Delta}_{1 n} \sqrt{\tilde{\omega}_{1 n}^{2}+\tilde{\Delta}_{1 n}^{2}} \sqrt{\tilde{\omega}_{2 n}^{2}+\tilde{\Delta}_{2 n}^{2}}}{\operatorname{det} 1},
\end{gathered}
$$

where

$$
\begin{aligned}
& \operatorname{det} 1=2(\sigma-1) \sigma \sqrt{\tilde{\omega}_{1 n}^{2}+\tilde{\Delta}_{1 n}^{2}}\left(\tilde{\Delta}_{1 n} \tilde{\Delta}_{2 n}+\tilde{\omega}_{1 n} \tilde{\omega}_{2 n}\right)- \\
& -[2(\sigma-1) \sigma+1]\left(\tilde{\omega}_{1 n}^{2}+\tilde{\Delta}_{1 n}^{2}\right) \sqrt{\tilde{\omega}_{2 n}^{2}+\tilde{\Delta}_{2 n}^{2}} .
\end{aligned}
$$

The solution for the second band is obtained from Eqs. 30-32) by replacing $1 \Longleftrightarrow 2$. In the Born limit one gets

$$
\begin{gathered}
\tilde{\omega}_{1 n}=\omega_{n}+\Gamma_{1} \frac{\sigma \tilde{\omega}_{2 n}}{\sqrt{\tilde{\omega}_{2 n}^{2}+\tilde{\Delta}_{2 n}^{2}}} \\
\tilde{\Delta}_{1 n}=\Delta_{1}+\Gamma_{1} \frac{\sigma \tilde{\Delta}_{2 n}}{\sqrt{\tilde{\omega}_{2 n}^{2}+\tilde{\Delta}_{2 n}^{2}}}
\end{gathered}
$$

i.e. the interband scattering mixes both bands. In the unitarity limit $\sigma \rightarrow 1(u \rightarrow \infty)$ the bands are decoupled, i.e.

$$
\begin{gathered}
\tilde{\omega}_{\alpha n}=\omega_{n}+\Gamma_{\alpha} \frac{\tilde{\omega}_{\alpha n}}{\sqrt{\tilde{\omega}_{\alpha n}^{2}+\tilde{\Delta}_{\alpha n}^{2}}} \\
\tilde{\Delta}_{\alpha n}=\Delta_{\alpha n}+\Gamma_{\alpha} \frac{\tilde{\Delta}_{\alpha n}}{\sqrt{\tilde{\omega}_{\alpha n}^{2}+\tilde{\Delta}_{\alpha n}^{2}}} .
\end{gathered}
$$

So, in this case the Anderson theorem is restored, i,e, the thermodynamic properties are impurity independent.

The latter result can be generalize to the case 


$$
\mathbf{u}^{N}=\left(\begin{array}{cc}
\alpha u & u \\
u & u_{22}
\end{array}\right)
$$

For $u \rightarrow \infty$ but $\alpha$ and $u_{22}$ finite, $\alpha$ and $u_{22}$ drop out from equations and the bands are decoupled with $\tilde{\omega}_{\alpha n}$ and $\tilde{\Delta}_{\alpha n}$ given by Eqs.35-36). At $T_{c}$ one has

$$
\begin{gathered}
\tilde{\omega}_{1 n}=\omega_{n}+\Gamma_{1} \operatorname{sign}\left(\omega_{n}\right) \\
\tilde{\Delta}_{1 n}=\Delta_{1}+\Gamma_{1}\left(\frac{\tilde{\Delta}_{1 n}}{\left|\tilde{\omega}_{1 n}\right|}+\frac{(1-\sigma) \tilde{\Delta}_{2 n}}{\left|\tilde{\omega}_{2 n}\right|}\right) .
\end{gathered}
$$

From $E q .(38)$ it is seen that in the unitarity limit, $\sigma \rightarrow 1$, the renormalized order parameters are decoupled and $T_{c}$ is unrenormalized. For $\sigma<1$ it can be easily shown that $T_{c}$ is reduced.

\section{SMALL ANISOTROPIC DEFECT IN ANISOTROPIC SUPERCONDUCTORS}

In what follows we consider the effect of a single impurity (small defect) with small scattering length $a$, which is supposed to be much smaller than the superconducting coherence length, $|a| \ll \xi_{0}$. Hence, the impurity can be considered as a localized perturbation, but with negligible renormalization of $\hat{\Delta}\left(\mathbf{p}_{F}, \mathbf{R}\right)$, giving rise to the quasiclassic equation, 20,21

$$
\begin{gathered}
{\left[\left(i \omega_{n}+e \mathbf{v}_{F} \cdot \mathbf{A}(\mathbf{R})\right) \hat{\tau}_{3}-\hat{\Delta}\left(\mathbf{p}_{F}, \mathbf{R}\right), \delta \hat{g}\left(\mathbf{p}_{F}, \mathbf{R}, \omega_{n}\right)\right]+i \mathbf{v}_{F} \nabla_{\mathbf{R}} \delta \hat{g}\left(\mathbf{p}_{F}, \mathbf{R}, \omega_{n}\right)=} \\
=\left[\hat{t}\left(\mathbf{p}_{F}, \mathbf{p}_{F}, \omega_{n}\right), \hat{g}_{i m t}\left(\mathbf{p}_{F}, \mathbf{R}, \omega_{n}\right)\right] \delta\left(\mathbf{R}-\mathbf{R}_{i m p}\right)
\end{gathered}
$$

Here, $\delta \hat{g}\left(\mathbf{p}_{F}, \mathbf{R}, \omega_{n}\right)=\hat{g}\left(\mathbf{p}_{F}, \mathbf{R}, \omega_{n}\right)-\hat{g}_{i m t}\left(\mathbf{p}_{F}, \mathbf{R}, \omega_{n}\right)$. The extra term proportional to $\delta\left(\mathbf{R}-\mathbf{R}_{i m p}\right)$ describes a jump in $\hat{g}\left(\mathbf{p}_{F}, \mathbf{R}, \omega_{n}\right)$ at the site $\mathbf{R}_{i m p}$ of the impurity (defect), while the intermediate Green's function $\hat{g}_{i m t}\left(\mathbf{p}_{F}, \mathbf{R}, \omega_{n}\right)$ describes the quasiclassic motion in the absence of impurity (defect) and it is the solution of Eq.(39) by putting the right-side to zero. $\hat{g}_{\text {imt }}\left(\mathbf{p}_{F}, \mathbf{R}, \omega_{n}\right)$ is normalized according to Eq.(2) $)$. The t-matrix is the solution of $E q$.(1) where $\hat{g}\left(\mathbf{p}_{F}, \omega_{n}\right)$ is replaced by $\hat{g}_{i m t}\left(\mathbf{p}_{F}, \mathbf{R}=\mathbf{R}_{i m p}, \omega_{n}\right)$. The change of the superconducting free-energy in the presence of a single impurity (defect) is given by 20,21 


$$
\delta F\left(\mathbf{R}_{i m p}\right)=N(0) T \sum_{n} \int_{0}^{1} d \lambda \int \frac{d^{2} \hat{k}_{F}}{4 \pi} \int d^{3} R T r\left[\delta \hat{g}\left(\mathbf{p}_{F}, \mathbf{R}, \omega_{n}\right) \hat{\Delta}_{b}\left(\mathbf{p}_{F}, \mathbf{R}\right)\right],
$$

where $\hat{\Delta}_{b}\left(\mathbf{p}_{F}, \mathbf{R}\right)$ and the vector potential $\mathbf{A}_{b}(\mathbf{R})$ are calculated in the absence of the impurity. The Green's function, $\delta \hat{g}\left(\mathbf{p}_{F}, \mathbf{R}, \omega_{n}\right)$, must be evaluated for an order parameter $\hat{\Delta}\left(\mathbf{p}_{F}, \mathbf{R}\right)=\lambda \hat{\Delta}_{b}\left(\mathbf{p}_{F}, \mathbf{R}\right)$.

In the following we study the consequences of anisotropic impurity scattering for three selected examples of inhomogeneous anisotropic superconductors.

\section{Bound states due to the anisotropic impurity}

Let us consider the local change of superconductivity in the presence of a single anisotropic impurity with the potential $v\left(\mathbf{s}, \mathbf{s}^{\prime}\right)$ given by Eq.(10) and analyze the impurityinduced quasiparticle bound state and the change in the free-energy $\delta F\left(\mathbf{R}_{\text {imp }}\right)$. By assuming that $2 \pi \bar{\sigma}_{i} \ll E_{F} / \Delta_{0}$, where $i=0,2$ and $\bar{\sigma}_{i}=v_{i}^{2} /\left(1+v_{i}^{2}\right)$, the t-matrix is given by the same expression as Eqs.(13 - 14), but with $\tilde{g}_{2}(n)$ is replaced by $g_{2}^{(0)}(n)$. The bound state energy $\omega_{B \text {,anis }}<\Delta_{0}$, which is due to the pair-breaking impurity effects, can be obtained as a pole of the $t$-matrix which gives

$$
\omega_{B, \text { anis }}=\Delta_{0} \sqrt{1-\bar{\sigma}_{p b}}
$$

where

$$
\bar{\sigma}_{p b}=\bar{\sigma}_{0} \bar{\sigma}_{2} \frac{\left(v_{0}-v_{2}\right)^{2}}{v_{0}^{2} v_{2}^{2}}
$$

In the unitarity limit for both channels, i.e. $v_{0} \gg 1, v_{2} \gg 1$ but $v_{2} / v_{0}$ finite, one has $\omega_{B, \text { anis }} \rightarrow \Delta_{0}$ contrary to the unitarity limit for the s-wave scattering $\left(v_{0} \gg 1, v_{2}=0\right)$ where $\omega_{B, i s o} \rightarrow 0$. However, the zero-energy bound state $\omega_{B \text {,anis }} \rightarrow 0$ appears when $v_{0} v_{2}=-1$, i.e. if one channel is in the unitarity limit the other one must be in the Born limit.

Due to the bound state there is a change (increase) of the free-energy $\delta F\left(\mathbf{R}_{i m p}\right) \equiv \delta F_{i m p}$. By solving Eq.(39) with $\hat{g}_{i m t}\left(\mathbf{p}_{F}, \mathbf{R}, \omega_{n}\right)$ given by Eq.(5) and $\hat{t}$ given by Eqs.11- 14) one gets $\delta F_{i m p}$ from Eq. (40)

$$
\delta F_{i m p}=T \sum_{n} \int_{0}^{1} d \lambda \bar{\sigma}_{p b} \frac{\lambda \Delta_{0}^{2} \omega_{n}^{2}}{\left[\omega_{n}^{2}+\lambda^{2} \Delta_{0}^{2}\right]\left[\omega_{n}^{2}+\omega_{B, a n i s}^{2}\right]}=
$$




$$
=2 T \ln \frac{\cosh \left(\Delta_{0} / 2 T\right)}{\cosh \left[\left(1-\sigma_{p b}\right)^{1 / 2} \Delta_{0} / 2 T\right]},
$$

where $\bar{\sigma}_{p b}$ is given in Eq.(42). It is seen that there is a loss in the condensation energy, $\delta F\left(\mathbf{R}_{i m p}\right)>0$, which is related to the pair-breaking effect of impurity. For $v_{0}=v_{2}$ such an impurity does not affect superconductivity and $\delta F\left(\mathbf{R}_{i m p}\right)=0$.

The obtained results tell us that in for angle-dependent impurity scattering even a strong impurity potential may have very weak effect on $T_{c}$, the bound state, and the free-energy of anisotropic and unconventional pairing. In that case the anisotropic pairing is robust in the presence of impurities.

\section{Pinning of single-vortex by a small anisotropic defect}

Because in HTS oxides strong correlations give rise to strong momentum-dependent charge scattering processes it is interesting to analyze the elementary-flux-pinning potential

of a small defect by using the approach of Thuneberg et al.,2,21, who showed that in s-wave superconductors the pinning energy of a small defect $\left(a \ll \xi_{0}\right)$ is dominated by scattering processes at the defect. It is proportional to the product of the scattering cross section and coherence length $\left(\propto a^{2} \xi_{0}\right)$, instead of (naively believed) $a^{3}$. The case of anisotropic pairing with s-wave impurities and near $T_{c}$ was recently studied in 22.

In what follows we study the effect of scattering anisotropy on the pinning energy of a small defect in an anisotropic superconductor at any temperature below $T_{c}$. We use the model potential given in Eq.(10) and assume that the vortex is placed at the defect. In order to calculate the elementary flux-pinning energy one has to solve the quasiclassical equations for various ballistic trajectories with $\mathbf{R}$-dependent the vector potential, $\mathbf{A}(\mathbf{R})$, and order parameter,

$$
\Delta_{b}\left(\mathbf{p}_{F}, \mathbf{R}\right)=\left|\Delta\left(\mathbf{p}_{F}, \mathbf{R}\right)\right| e^{i \theta} Y(\theta)
$$

In the gauge where $\theta$ is the angle with respect to the $\mathrm{X}$-axis then $\mathbf{A}(\mathbf{R})$ has no radial component. The solution of Eq.(39) requires for a realistic vortex numerical calculations. For a qualitative discussion we will adopt a simplified vortex model20,21 which neglects the 
suppression of the order parameter in the vortex core and sets $\left|\Delta_{b}\left(\mathbf{p}_{F}, \mathbf{R}\right)\right|=\Delta_{0}\left(\mathbf{p}_{F}\right)$, i.e. independent of $\mathbf{R}$. Hence, the order parameter along a trajectory passing through the vortex center has constant magnitude but its phase changes abruptly by $\pi$ when going through the vortex core. This "zero-core model" gives the right order of magnitude of the pinning energy, $\delta F_{\text {pin }}\left(\mathbf{R}_{i m p}\right)$, when compared with the numerical calculations 21 . In order to calculate $\delta F_{p i n}\left(\mathbf{R}_{i m p}\right)$ two quantities are needed: $\hat{g}_{i m t}\left(\mathbf{p}_{F}, \mathbf{R}=\mathbf{R}_{i m p}, \omega_{n}\right) \equiv \hat{g}_{v}\left(\mathbf{p}_{F}, \mathbf{R}=\mathbf{R}_{i m p}, \omega_{n}\right)$ in the presence of the zero-core vortex and the impurity $t$-matrix calculated with the Green's function, $\hat{g}_{v}\left(\mathbf{p}_{F}, \mathbf{R}=\mathbf{R}_{i m p}, \omega_{n}\right)$, of the zero-core model. The solution is straightforward 21 and gives

$$
\hat{g}_{v}\left(\mathbf{p}_{F}, \mathbf{R}=\mathbf{R}_{i m p}, \omega_{n}\right)=\frac{1}{\omega_{n}}\left[\left(-\Delta_{2} \hat{\tau}_{1}+\Delta_{1} \hat{\tau}_{2}\right) Y(\theta)+\left(-i \alpha_{n}\right) \hat{\tau}_{3}\right]
$$

and

$$
\hat{t}\left(\mathbf{p}_{F}, \mathbf{p}_{F}, \omega_{n}\right)=t_{3} \hat{\tau}_{3}=-i \gamma_{u} \alpha_{n} \omega_{n}\left[\frac{\bar{\sigma}_{0}}{\omega_{n}^{2}+\bar{\sigma}_{0} \Delta_{0}^{2}}+\frac{\bar{\sigma}_{2}}{\omega_{n}^{2}+\bar{\sigma}_{2} \Delta_{0}^{2}}\right] \hat{\tau}_{3}
$$

Here, $\alpha_{n}=\sqrt{\omega_{n}^{2}+\Delta_{0}^{2}}$. Eq.(39) can be solved by the Fourier (or Laplace) transform which gives the expression for the pinning free-energy

$$
\begin{gathered}
\delta F_{p i n}=\delta F_{p i n}^{(s t i f f)}\left(\bar{\sigma}_{0}, \bar{\sigma}_{2}\right)+\delta F_{p i n}^{(p b)}\left(\sigma_{p b}\right) \\
\delta F_{p i n}^{(s t i f f)}=-2 T \ln \left\{\cosh \frac{\sqrt{\bar{\sigma}_{0}} \Delta_{0}}{2 T} \cdot \cosh \frac{\sqrt{\bar{\sigma}_{2}} \Delta_{0}}{2 T}\right\} \\
\delta F_{p i n}^{(i m p)}=-2 T \ln \frac{\cosh \left(\Delta_{0} / 2 T\right)}{\cosh \left[\left(1-\bar{\sigma}_{p b}\right)^{1 / 2} \Delta_{0} / 2 T\right]} .
\end{gathered}
$$

Eqs.47-49) imply that, $\delta F_{\text {pin }}<0$, and the vortex is attracted (pinned) by the defect. A comparison of Eq.47) with the corresponding results for $s$-wave superconductors with an s-wave scattering potential shows, that in the former case two additional terms are present. The first one, depending on $\bar{\sigma}_{2}$, appears also in s-wave superconductors with anisotropic scattering accounted for. In fact $\delta F_{\text {pin }}^{(\text {stiff })}$ describes the reduction of the superconducting stiffness in the presence of impurities. For instance near $T_{c} E q .(48)$ gives 


$$
\delta F_{p i n}^{(s t i f f)}=-\left(\bar{\sigma}_{0}+\bar{\sigma}_{2}\right) \cdot \frac{\Delta_{0}^{2}(T)}{4 T_{c}} \approx-7.6 \frac{\bar{\sigma}_{0}+\bar{\sigma}_{2}}{v_{F}^{2}} \xi_{0}^{2} \cdot T_{c} \Delta_{0}^{2}(T),
$$

and $\delta F_{\text {pin }}^{(s t i f)}$ is proportional to the total scattering amplitude $\bar{\sigma}_{0}+\bar{\sigma}_{2}$. For vortex far away from the impurity there is loss in the condensation energy $\delta F_{p i n}^{(p b)}\left(\bar{\sigma}_{p b}\right)$ due to pair-breaking effect of the impurity, i.e. $\delta F_{p i n}^{(p b)}\left(\bar{\sigma}_{p b}\right)=-\delta F_{i m p}\left(\bar{\sigma}_{p b}\right)$ where $\delta F_{i m p}\left(\bar{\sigma}_{p b}\right)$ is given by $E q$. (43). Therefore this part enters in Eq.47) with the negative sign, thus increasing the pinning energy when vortex is sitting on the defect and stabilizing it additionally. Near $T_{c}$ one has

$$
\delta F_{p i n}^{(p b)}\left(\sigma_{e f f}\right)=-\bar{\sigma}_{p b} \cdot \frac{\Delta_{0}^{2}(T)}{4 T_{c}}
$$

For the s-wave scattering only, $v_{2}=0$, one has $\bar{\sigma}_{2}=0, \bar{\sigma}_{p b}=\bar{\sigma}_{0}$, and the pair-breaking effect is maximal while the condensation energy is gained maximally for vortex sitting on the defect. However, for, $v_{2}=v_{0}$ the pair-breaking of impurity is absent $\bar{\sigma}_{p b}=0$ and $\delta F_{p i n}^{(p b)}=0$, i.e. in this case the pinning by the small defect is similar to that in s-wave superconductors.

The physical picture of the vortex pinning by small defect given above is based on the known results based on the microscopic derivation of the Ginzburg-Landau equations in the presence of impurities. An explanation based on the quasiclassical approach is given in 20,21 and we briefly discuss it in order to develop an intuition for the case of a double-vortex pinning, which is studied below. Because the order parameter changes its phase by $\pi$ along the trajectories across the vortex core it leads to the phase change of $\hat{g}_{v}\left(\mathbf{p}_{F}, \mathbf{R}, \omega_{n}\right)\left(\equiv \hat{g}_{i m t}\left(\mathbf{p}_{F}, \mathbf{R}, \omega_{n}\right)\right)$ on the distance $\xi_{0}$, thus causing a cost in the condensation energy, i.e. the maximal increase of the free-energy. Note, the function $\hat{g}_{v}\left(\mathbf{p}_{F}, \mathbf{R}, \omega_{n}\right)$ describes the quasiclassical motion of particles (or pairs) along trajectories across the vortex core where the maximal phase change $(\pi)$ occurs. In the presence of defect the motion of particles is described by the function $\hat{g}\left(\mathbf{p}_{F}, \mathbf{R}, \omega_{n}\right)$ which contains scattering of particles to new directions where the phase change (mismatch) is less than $\pi$ and it costs less condensation energy. Therefore the vortex is attracted to the defect because scattering helps superconductivity to sustain abrupt changes in the order parameter. The latter explains the contribution $\delta F_{\text {pin }}^{(s t i f)}$, while in the anisotropic superconductors, due to pair-breaking effects 
of impurities, there is a gain of condensation energy $-\delta F_{i m p}\left(\sigma_{e f f}\right)$ for vortex sitting on the defect.

\section{Pinning of double-vortex by small defect}

We extend the calculations in 21 to the pinning of multiply-quantized vortices on small defects. First, a s-wave superconductor is considered and we put the question - is it possible to pin the double-flux-vortex $\left(\Phi=2 \Phi_{0}\right)$ by the small defect, which is for simplicity characterized by the parameter $\bar{\sigma}_{0}$ for s-wave scattering only? The "zero-core model" is assumed again. In that case the order parameter can be parametrized in the form

$$
\Delta_{b}\left(\mathbf{p}_{F}, \mathbf{R}\right)=\left|\Delta\left(\mathbf{p}_{F}, \mathbf{R}\right)\right| e^{2 i \theta}
$$

For particle motion across the double-vortex core the order parameter does not change phase and in that case the solutions for $\hat{g}_{i m t}\left(\mathbf{p}_{F}, \mathbf{R}=\mathbf{R}_{i m p}, \omega_{n}\right)\left(\equiv \hat{g}_{2 v}\left(\mathbf{p}_{F}, \mathbf{R}=\mathbf{R}_{i m p}, \omega_{n}\right)\right)$ and for $\hat{t}\left(\mathbf{p}_{F}, \mathbf{p}_{F}, \omega_{n}\right)$ are given by

$$
\hat{g}_{2 v}\left(\mathbf{p}_{F}, \mathbf{R}=\mathbf{R}_{i m p}, \omega_{n}\right)=\frac{i}{\alpha_{n}}\left[\Delta_{1} \hat{\tau}_{1}+\Delta_{2} \hat{\tau}_{2}+\left(-\omega_{n}\right) \hat{\tau}_{3}\right]
$$

and

$$
\hat{t}\left(\mathbf{p}_{F}, \mathbf{p}_{F}, \omega_{n}\right)=t_{3} \hat{\tau}_{3}=-i \omega_{n} \alpha_{n} \gamma_{u} \frac{\bar{\sigma}_{0}}{\omega_{n}^{2}+\tilde{\sigma}_{0} \Delta_{0}^{2}} \hat{\tau}_{3}
$$

where $\tilde{\sigma}_{0}=\bar{\sigma}_{0} / v_{0}^{2}$. Then by solving Eq.(39) and by using Eq.(40) and this solutions one obtains the pinning energy of the double-vortex within the zero-core model

$$
\delta F_{2 v, p i n}=2 T \sum_{n} \int_{0}^{1} d \lambda \frac{\lambda \Delta_{0}^{2} \omega_{n}^{2} \bar{\sigma}_{0}}{\alpha_{n}^{2}\left[\omega_{n}^{2}+\tilde{\sigma}_{0} \lambda^{2} \Delta_{0}^{2}\right]}>0 .
$$

The main conclusion coming out from $E q$. (55) is that because $\delta F_{p i n}>0$ the double-vortex in s-wave superconductors is repelled from the defect - i.e. the zero-core double-vortex can not be pinned. Contrary to the single-vortex, where the defect scatters particles to new directions where the phase change is smaller, in the case of double-vortex the particles are scattered to directions where the phase change is larger. However, it might be that the above obtained results are an artefact of the "zero-core model", where there is no suppression of 
the superconducting order due to the vortex core, and numerical calculations are required for a realistic double-vortex structure23.

In the case of an unconventional pairing, like that in Section II.1, the order parameter is given by $\Delta_{b}\left(\mathbf{p}_{F}, \mathbf{R}\right)=\left|\Delta\left(\mathbf{p}_{F}, \mathbf{R}\right)\right| \exp (2 i \theta) Y(\theta)$ and the t-matrix contains also terms $t_{1}, t_{2} \neq 0$ leading to a decrease of the jump $\left[\hat{t}\left(\mathbf{p}_{F}, \mathbf{p}_{F}, \omega_{n}\right), \hat{g}_{i m t}\left(\mathbf{p}_{F}, \mathbf{R}, \omega_{n}\right)\right]$ in Eq. (39). In this case the pinning energy contains the additional term (gain in energy) due to the pairbreaking effect of the impurity, $-\delta F_{i m p}$, i.e. $\delta F_{p i n}=\delta F_{2 v, p i n}-\delta F_{i m p}$. Since $\delta F_{2 v, p i n}$ is less positive (repulsive) than for a s-wave superconductor in $E q$. (55), and because $-\delta F_{\text {imp }}<0$ one can happen that $\delta F_{\text {pin }}<0$ and even the zero-core double-vortex can be pinned by the defect. A realistic calculation of $\delta F_{\text {pin }}$ for anisotropic superconductors with anisotropic scattering of single and double-vortex will be discussed elsewhere 23 .

In conclusion the anisotropic impurity scattering gives rise to new qualitative effects in unconventional and anisotropic superconductors, where for instance it "screens" the strength of the scattering in some quantities (like $T_{c}$ - robustness of pairing, bound states, pinning, etc.) even in the unitarity limit. It seems that this situation is partly realized in HTS oxides where the d-wave pairing is robust in the presence of even very strong impurity scattering. In two-band models nonmagnetic impurities do not affect thermodynamic properties of swave superconductors in the unitarity limit for the interband scattering, contrary to the Born limit, i.e. in this case the Anderson theorem is restored in the unitarity limit.

\section{Acknowledgments}

M. L. K. thanks Dierk Rainer for valuable discussions, suggestions and for careful reading and correcting the manuscript. M. L. K. acknowledges gratefully the support of the Deutsche Forschungsgemeinschaft through the Forschergruppe "Transportphänomene in Supraleitern und Suprafluiden". O.V. D. thanks Nils Schopohl for valuable discussions and support. 


\section{REFERENCES}

${ }^{1}$ M. L. Kulić, review, preprint University Bayreuth, 1998

2 J. Sauls, Adv. Phys. 43, 113 (1994)

${ }^{3}$ A. G. Sun, L. M. Paulius, D. A. Gajevski, M. B. Maple and R. C. Dynes, Phys. Rev. B50, 3266 (1994); A. G. Sun, D. A. Gajevski, M. B. Maple and R. C. Dynes, Phys. Rev. Lett., 72, 2267 (1994); S. K. Tolpygo, J.-Y. Lin, M. Gurvitch, S. Y. Hou, J. M. Philips, Phys. Rev. B53, 12454 (1996); ibid 12462 (1996)

${ }^{4}$ S. V. Pokrovsky and V. L. Pokrovsky, Phys. Rev. B54, 13275 (1996); in fact the theory is given for the first time in P. C. Hohenberg, Sov. Phys. JETP, 18, 834 (1964)

${ }^{5}$ M. L. Kulić, V. Oudovenko, Solid State Comm. 104, 731 (1997)

${ }^{6}$ A. J. Millis, S. Sachdev and C. M. Varma, Phys. Rev. B 37, 4975 (1988); G. Harán, A. D. S. Nagi, Phys. Rev. B54, 15463 (1996)

7 T. Hotta, J. Phys. Soc. Jpn. 62, 2744 (1993); L. S. Borkowski, P. J. Hirschfeld, Phys. Rev. B49, 15404 (1994); R. Ferenbacher, M. Norman, Phys. Rev. B50, 3495 (1994); Y. Sun, K. Maki, Phys. Rev. B51, 6059 (1995)

${ }^{8}$ M. L. Kulić and R. Zeyher, Phys. Rev. B49, 4395 (1994); R. Zeyher and M. L. Kulić, Phys. Rev. B53, 2850 (1996); ibid B54, 8985 (1996)

${ }^{9}$ U. Hofmann, J. Keller, M. L. Kulić, Z. Phys. B - Condensed Matter 81, 25 (1990)

${ }^{10}$ R. Gajić,. J. Keller, M. L. Kulić, Solid State Comm. 76, 731 (1990)

11 A. A. Golubov, I. I. Mazin, Phys. Rev. B 55, 15146 (1997)

${ }^{12}$ R. Combescot, X. Leyronas, Phys. Rev. Lett. 75, 3732 (1995)

${ }^{13}$ G. Eilenberger, Z. Phys. 180, 32 (1968)

${ }^{14}$ A. I. Larkin, Yu. N. Ovchinnikov, Zh. Eksp. Teor. Fiz. 55, 2262 (1968) (Sov. Phys.-JETP 
26, $1200(1968))$

${ }^{15}$ J. W. Serene, D. Rainer, Phys. Rep. 101, 221 (1983)

${ }^{16}$ J. R. Schrieffer, X.-G. Wen, S. -C. Zhang, Phys. Rev. B 39, 11663 (1989)

${ }^{17}$ D. Rainer, in Progress in Low Temperature physics, ed. D. F. Brewer, North Holland 1986, Ch. 4, p. 415

${ }^{18}$ G. Preosti, H. Kim, P. Muzikar, Phys. Rev. B 50, 1259 (1994)

${ }^{19}$ S. Haas, A. V. Balatsky, M. Sigrist, T. M. Rice, Phys. Rev. B 56, 5108 (1997)

${ }^{20}$ E. V. Thuneberg, J. Kurkijärvi, D. Rainer, Physica 107 B, 43 (1981)

${ }^{21}$ E. V. Thuneberg, J. Kurkijärvi, D. Rainer, Phys. Rev. Lett. 48, 1853 (1982); Phys. Rev. B 29, 3913 (1984)

${ }^{22}$ M. Friesen, P. Muzikar, Phys. Rev. B 53, R11953 (1996)

${ }^{23}$ M. Endres, M. L. Kulić, D. Rainer, ongoing work 